Rapid Reviews COVID-19

\title{
Review 1: "Binding Profile Assessment of N501Y: a More Infectious Mutation on the Receptor Binding Domain of SARS-CoV-2 Spike Protein"
}

\section{Arieh Warshel ${ }^{1}$}

${ }^{1}$ Professor, USC: University of Southern California, Chemistry, USA

Published on: Mar 15, 2021

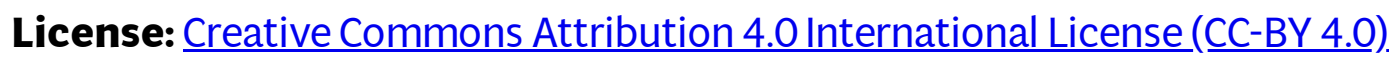




\section{$\underline{\text { RR:C19 Evidence Scale rating by reviewer: }}$}

- Potentially informative. The main claims made are not strongly justified by the methods and data, but may yield some insight. The results and conclusions of the study may resemble those from the hypothetical ideal study, but there is substantial room for doubt. Decision-makers should consider this evidence only with a thorough understanding of its weaknesses, alongside other evidence and theory. Decisionmakers should not consider this actionable, unless the weaknesses are clearly understood and there is other theory and evidence to further support it.

$* * * * * * * * * * * * * * * * * * * * * * * * * * * * * * * * * * * * * * *$

\section{Review:}

The paper addresses an important and current problem but conveniently attempts to ignore a study that already addressed the same problem more than nine months ago. The importance of the N105 mutants has already been predicted in Critical Differences between the Binding Features of the Spike Proteins of SARS-CoV-2 and SARS-CoV, C. Bai and A. Warshel: J. Phys. Chem. B, 124, 5907-12 (2020). This work also generated a binding profile for other mutant. Obviously the present paper must clearly discuss the previous work, clarifying its findings before trying to publish a similar finding. 